\title{
Gluten-free Diet - Tough Alley in Torrid Time
}

\author{
Lerner Aaron ${ }^{1,2, *}$, Matthias Torsten ${ }^{2}$ \\ ${ }^{1}$ B. Rappaport School of Medicine, Technion-Israel Institute of Technology, Haifa, Israel \\ ${ }^{2}$ AESKU.KIPP Institute, Wendelsheim, Germany \\ *Corresponding author: aaronlerner1948@gmail.com
}

\begin{abstract}
Dietitians play a pivotal role in the education, follow-up and navigation of the gluten-free diet for patients affected by celiac disease. Since gluten withdrawal is the cornerstone of celiac disease therapy, and since various future therapeutic strategies, are not yet on the market, the patients relay heavily on the registered dietitian nutritionists (RDNs) advice and service to cope with the gluten-free diet tough alley. Unfortunately, gluten withdrawal, nowadays, represent also a torrid time. The actual surge in incidence, wheat content, gluten intake, celiac disease-related T-cell stimulatory epitopes in wheat, usage in the processed food industries, nutritional deficiencies, changing phenotype and the fact that gluten is potentially detrimental to humankind health, make the RDNs role more complex, difficult and challenging. The present review expands on the gluten-free diet related tough alley in torrid time, which the registered dietitian nutritionists are facing when dealing with gluten-sensitive patients.
\end{abstract}

Keywords: gluten-free diet, celiac disease, dietitians, compliance, adherence

Cite This Article: Lerner Aaron, and Matthias Torsten, "Gluten-free Diet - Tough Alley in Torrid Time." International Journal of Celiac Disease, vol. 5, no. 2 (2017): 50-55. doi: 10.12691/ijcd-5-2-4.

\section{Introduction}

Dietitians play a pivotal role in the education, follow-up and navigation of the Gluten-free diet (GFD) for patients affected by celiac disease (CD). Since Gluten withdrawal is the cornerstone of CD therapy, and since various future therapeutic strategies, are not yet on the market [1], the patients relay heavily on the dietitians' advice and service to cope with the GFD tough alley. Numerous studies evaluated the Gastroenterologist-CD patient interactions but very few evaluated the bilateral dietitian-CD patient axis, during their interactions. More so, the patients' perspective toward the dietitians was explored in few studies, reporting lack of expertise among registered dietitian nutritionists (RDNs) [2,3,4]. But the other way around, the dietitian's perspectives toward the patients was scarcely investigated. This is the raison why Gieger et al should be congratulated for measuring RDN self-reported CD knowledge and preferences for resources intendant for self and patient educations [5]. Based on 405 RDNs from 7 states in the USA, 35 item internet-based surveys, they concluded that RDNs may need more reinforcement on identification and treatment of nutritional deficiencies of $\mathrm{CD}$ management. In order to set up the stage for discussion, the present review will zoom on two GFD topics: why it is a tough alley and why we are fronting a torrid time.

\section{GFD, Why Tough Alley}

\subsection{GFD is the Only Effective Therapy for CD, for now, but on the Other Hand, Compliance to the Restrictive Diet Embeds Multiple Difficulties that Compromise Its Adherence}

GFD imposes a large number of restrictions on the patient, having both lifetime and financial implications. Table 1 summarizes the problematic aspects of gluten restriction that were associated with lower compliance. Those difficulties are the diving force to develop alternative nutritional and non-nutritional therapies for CD [1].

Table 1. The difficulties to follow gluten-free diet

\begin{tabular}{|l|l|}
\hline Low/poor availability of GF products & More expensive \\
\hline Social pressure for gluten containing diet & Inconsistent, confusing, incorrect or lack of labeling \\
\hline Low palatability & Cross contamination \\
\hline Inconvenient & Lack of consensus about the minimal amount of gluten allowed \\
\hline Compromised texture & Lack of standardization of gluten content determination \\
\hline GFD poses nutritional deficiencies and unbalanced diet & Persistent symptoms on GFD \\
\hline Limited availability of dietary counseling & Individual gluten sensitivity \\
\hline Gluten found in 70\% of manufactured food products & Lack of manufacturing regulations in many countries \\
\hline Anger, fear and anxiety after the diagnosis & Dissatisfaction with information provided by professionals \\
\hline $\begin{array}{l}\text { Inaccurate information from food stores, alternative practitioners, family, friends } \\
\text { and other resources }\end{array}$ & Insufficient or misinformation on GFD \\
\hline
\end{tabular}




\subsection{GFD is not Standardized and Its CD Customers are Quite Confused}

The customers are exposed to inaccurate information from the books, periodicals, internet, social media and smart phone applications [6,7]. The cellulars' app is expanding and it becomes difficult to monitor, review, and compare them for their reliability and accuracy $[8,9,10,11,12]$. This problem is expanded due to the fact that the internet is the preferred resource for RDNs self and patients educations [5,13]. More than 20 on-line sources of information on CD and GFD are available [14].

On top of it, CD is changing constantly, in respect to its geo-epidemiology, incidence, epidemiology, presenting symptoms, age of diagnosis, mode of diagnosis, serological biomarkers, monitoring of GFD compliance, understanding its pathophysiology and the associated environmental factors, newly evolving high-risk populations and indications for serological screen. All those aspects oblige the health care personal, including the RDNs to update and keep up with the dynamic and novel $\mathrm{CD}$ rapidly increasing information. The $\mathrm{CD}$ patients are between the gastroenterologists, family practitioners and the RDNs that don't coordinate the information delivered to a specific patient or the child's parents. So, sometimes the customer is confused and stressed, resulting in frustration that might impact is GFD compliance.

Even the definition of $\mathrm{CD}$ is evolving from the first consensus definition of CD published in Acta Paediatrica in 1970 [15] to the last Oslo definitions for CD and related terms, published online in 2012 [16]. Concerning the last one, since 2012, there is more, new information that might impact the definitions and terms. Even the diagnostic gold standard of duodenal biopsy's histology, is debatable [17] and recently the intraepithelial lymphocyte count threshold provoked definition exchanges between the experts $[18,19,20]$.

Another aspect for confusion for the treating physicians, RDNs and mainly the end customers is the threshold for gluten contamination in gluten-free products. A wide range exists in the literature, going from $<10$ to $200 \mathrm{ppm}$ (100ppm/day=30 mg gluten) $[21,22,23,24]$. More provocative is the case report showing that "A milligram of gluten a day keeps the mucosal recovery away" [25], raising the question: Is it the compliance, not milligrams of gluten that is essential in the treatment of celiac disease? [26]. Notably, a separate threshold for naturally GFD and rendered gluten-free products was suggested $(<20,100$ ppm, respectively) [23].

The serological prediction, screening, diagnosis, monitoring GFD compliance is not well studied and fare away from standardization. The following is the summary of limitations that are important to clinicians, laboratory staff, dieticians, researchers and others dealing with gluten-sensitive conditions, concerning IgA-TG2 antibodies [27]: The diversity of available test kits, the discrepancy between selective scientific validation, at the bench or bed environment and the fact that they reflect the activity of reactive but not innate immunity, or the intestinal inflammatory state of the CD intestinal mucosa. Furthermore, their performance is age-dependent, not reliable in monitoring disease activity on GFD in CD treated patients and lack of data on the impact of the serological activities on long-term prognosis, complications, extra-intestinal manifestations or autoimmunity genesis or progression. It should be noted that the debate whether to use only IgA-TG2 or to combine a complementary diagnostic autoantibody, to improve diagnosis and followup performance, is as yet unresolved. On top of all, a discrepancy between manufacturer's cut-off and receiver operating characteristic plot derived cut-off values modifies decision thresholds of the kits assays. More so, Kappa analysis demonstrates variable degrees of agreement. Sometime this variability can reach $75 \%$. In young children and even in older ones, seropositivity of IgA-TG2, may be a transient phenomenon, and not necessarily predictive for $\mathrm{CD}$ development. Several authors have raised questions regarding the diagnostic performance of IgA-TG2 antibodies in routine clinical practice. Several strategies have been suggested to improve performance or interpretation of the test, but these have not gained wide laboratory application [27].

At the end of the day, those limitations, difficulties, discrepancies, drawbacks, confusion, lack of appropriate definitions and standardization, numerous false positive and false negative of the gold standard serological marker [27] and lack of peer reviewed reliable information sources, impact the physician and RDNs relations with the end customers and are potentials for delayed or misdiagnosis, mal treatment and lower GFD compliance. As said, GFD in CD is neither a solved problem nor a "closed case".

\subsection{GFD Needs are Age-dependent}

Throughout a person's life, certain events will occur which is of particular importance and is considered as a turning point in their lives, so is the diagnosis of CD and the age-dependent nutritional habits. Food habits, nutrient needs and intakes differ between infants, children, adolescents, adults and the elderly, and so is their GFD when affected by CD. Dietitians should take the opportunity to reinforce a generally healthy diet when providing information about the GFD, taking in account the age related habits and needs. More so, since pediatric and adult dietician's consultations are two different domains and GFD is very age dependent, professional sub-specialization should be encouraged.

Infancy: A lot was published on the importance of the interaction between caregiver and child during feeding and the influence of parenting style on dietary habit formation. The effects of nutritional counseling of pediatric RDNs for the infant diet focused on lactating, complementary feeding and transition to child food is important, potentially impacting life-term food habits. Much more when the infant is diagnosed as CD. Since the first encounter of gluten happens on complementary food (cereals, oat, soft bakery products) the dietitian counseling should start early after the diagnosis and implement GFD as smooth and pleasant as possible to the baby and his parents.

Childhood: Skipping breakfast, low intake of fruit and vegetables, high consumption of both sugar-sweetened beverages and energy dense snacks may be dietary determinants of morbidity [28]. Schools and kinder gardens are very involved in initiatives of promotion of 
good dietary habits. Intervention programs to improve children's eating and leisure habits are frequently implemented and supported by the institutions and involvement of the families [29]. However, when a child has to go on a GFD, those supports are not enough. The role of the RDNs is crucial and indispensable in the initiation, maintenance and follow-up of the CD child. Since adolescence is the most vulnerable period for less compliance to GFD, the following paragraph will be expanded.

Adolescence: Adolescence is a period of fast psychological development, rapid growth and the appearance of secondary sex characteristics. Due to rapid physical growth of adolescents, physiological activities are increased and they need more energy to meet increasing demands in comparison to previous developmental period. Low meal frequency, skipping breakfast, and a high consumption of sugar sweetened beverages and unregular family meals are some of the characteristic nutritional habits during the adolescence period. However, the available data seem to show that the tendency in the adolescent population worldwide is to increase those dietary factors related with obesity development. And obesity is one of the newly reported phenotype in $\mathrm{CD}$, on presentation and during GFD [30]. Adolescents eat half of the recommended amount of fruit and vegetables and less than two-thirds of the recommended amount of milk and milk products but consume more meat and meat products, fats, and sweets than recommended [31].

Noncompliance with the GFD is often reported among adolescents with CD. However, knowledge is limited regarding their own perspectives and experiences of managing the disease and the prescription of the restricted diet. The probability of compliance with the GFD is comprised by insufficient knowledge of significant others, problems with the availability and sensory acceptance of gluten-free food, insufficient social support and their perceived dietary deviance (see Table 1). Three different approaches that can change the RDNs counseling and follow-up the GFD can be distinguished: compliers, occasional non-compliers, and non-compliers. Coping strategy depends on multiple factors, the main being a personal, trustful and sympathetic approach [32].

ELDERLY: A life-long GFD is restrictive, increasing the burden of the illness and impairing life quality. In the elderly, where adaptation to dietary habits are life-long deeply imbedded and hard to disrupt, the diet may not be tolerated. Limited financial and social resources, decreased mobility, difficult access to gluten-free products, impaired vision, cognitive decline and poor nutritional intakes are some of the problems that the elderly may confront [33]. Despite the above problematic aspects, it should be noted that $\mathrm{CD}$ affected elderlies might benefit and have dramatic improvement on gluten restrictive nutritional therapy $[33,34,35]$. Recently, it was shown that the compliance to GFD is very good, iron deficient anemia is restored, other nutritional deficiencies and bone mineral density ameliorate, gastrointestinal symptoms, though subtle, were alleviated and histological or serological recovery was virtually complete, in screen-detected older CD patients $[34,35]$. Older age should not be a barrier to dietary therapy. Still, due to special needs and increased morbidity of the older CD patients, a comprehensive, multidisciplinary and holistic approach of the DRNs will improve their outcome. Since often confronted with multiple supplemented or restrictive diets (high caloric, high iron and vitamin sources, high fiber, low sodium, low simple sugars, high or low proteins etc.,), gluten restriction, in the elderly, make the RDNs job much more complicated, though challenging.

\section{GFD, Why Torrid Time}

The CD population is facing nowadays torrid time due to the actual surge in incidence, wheat content, gluten intake, CD-related T-cell stimulatory epitopes in wheat, usage in the processed food industries, nutritional deficiencies, changing phenotype and the fact that gluten is potentially detrimental to humankind health [36-45].

\subsection{Increase in Gluten Wheat Content}

During the last centuries of our modern age, extensive genetic natural selection, due to changing environment and men-originated breeding manipulations, selective advantage of wheat toward improved grains number and wheat survival and adaptation, occurred. This evolutionary progression resulted in more than 20 thousand species of wheat known nowadays. The process was accompanied by enrichment of gluten content in the wheat. In fact, multiple recent studies documented the increased CD epitopes along the process of gluten gene enrichment in the passage of ancient diploid to modern tetraploid and hexaploid wheat species $[46,47,48,49]$. Today, gluten proteins comprise gliadins and glutenins, which are present in approximately equal amounts and form $80 \%$ of the total storage protein content in the wheat kernel. On the contrary, the ancient wheat was low in gluten, averaging $5-10 \%$ of the protein content $[49,50]$.

\subsection{Increase in Gluten Intake}

Wheat growing is larger than for any other crop and the world trade in wheat is greater than that for all other crops combined. Along with rice, wheat is the world's most favored staple food. In addition, wheat is the most important source of carbohydrate in a majority of countries. In the 20th century global wheat output expanded by about 5 -fold. Since the fifties there has been a dramatic ten-fold increase in the rate of wheat yield improvement per year, and this has become the major factor associated with increases in global wheat production. It goes without saying that the surge in wheat sales results in a parallel increase in gluten consumption and usage, impacting the gluten sensitive populations [39].

\subsection{Increase in CD-related T-cell Stimulatory Epitopes in Wheat}

Multiple recent studies documented the increased CD epitopes along the process of gluten gene enrichment in the passage of ancient diploid to modern tetraploid and hexaploid wheat species $[46,47,49]$. Once again, this is an additional gluten toxicity, adding to the actual torrid time for the GFD consumers. 


\subsection{Increased Usage by the Processed Food Industry}

The food industry is expanding towards new technologies, introducing additives and ingredients to the processed food, thus changing the composition and antigenicity of modern food products [38,39]. Most recently, based on scientific and internet originated literature, gluten was found to be a major food additive in the industrial processed food industries with a net increase in usage of $1.8+/-0.4 \% /$ year [39]. Since in many of Western populations the majority of the calories are coming from processed food (63\% in USA) and since gluten is one of the major nutrients added to the processed food, its continuous increased consumption represents an extra load on populations that are gluten-sensitive.

\subsection{Increased Incidence of $C D$ and Associated Autoimmune Disease}

Those increases represent an extra-cargo on the RDNs when dealing with GFD implementation, since they face more CD patients and have to deal with additional associated autoimmune diseases and morbidities. In fact, reviewing available literature, it can be deduced that incidences of CD have increased significantly over the last 60 years. In Canada, Scotland, and Spain the \%/year incidence surges were highest while in Estonia, USA and New Zealand it was the lowest [37]. Interestingly, the CD trend parallels the surge in many other autoimmune diseases, not only in the gastrointestinal domain [36]. A well-known observation is the age-dependent increase of autoimmune disease in the CD patients and even in their close relatives [51,52,53].

\subsection{Increased Nutritional Deficiencies}

The list of nutritional deficiencies in naïve and treated $\mathrm{CD}$ patients is increasing constantly. Not only the malabsorptive or the nutritional habits, but GFD itself is associated with numerous nutritional deficiencies like: low B vitamins, folate, calcium, vitamin D, iron, zinc, magnesium, carnitine and fiber $[5,54,55,56]$.

\subsection{Changing Phenotype toward Extra-intestinal Manifestations}

It has been shown that the classical intestinal clinical picture of malnutrition, chronic diarrhea and nutritional deficiencies are disappearing and extra-intestinal presentations are emerging. Skin, endocrine, skeletal, muscular, rheumatic, pulmonary, hepatic, renal, hematological, thromboembolic, nutritional, gynecological, fertility, dental, neuronal and behavioral abnormalities are often described [51,57,58], More so, nowadays, we are witnessing an epidemiological shift in the disease phenotype towards a more advanced age and an increased prevalence of latent, hypo symptomatic or asymptomatic behavior. Once again it represents an extra knowledge and management load on the RDNs that has to cope with the changing presentations and expending extra-intestinal remote organ conditions' morbidities.

\subsection{Gluten is Potentially Detrimental to Humankind}

Finally, dealing with GFD implementation, not only in $\mathrm{CD}$ but in additional gluten related conditions and the modern trend of GFD, it seems to us that the RDNs should be updated on numerous side effects that gluten might induce. It appears, based on animal models, immortalized cell-lines and ex-vivo studies that gluten has various detrimental effects. It impacts the microbiome, increases intestinal permeability. Gluten is immunogenic, cytotoxic, pro-inflammatory and activates the immune system, increases apoptosis, decreasing viability and differentiation, acts as pro-inflammatory enhancer of oxidative stress and impacts epigenetic processes. Interestingly, gluten withdrawal in certain non-celiac autoimmune diseases may be beneficial to minimize gluten's disadvantageous effects. (Personal communication, unpublished data). It should be emphasized that not enough studies where performed on gluten side effects in human and the unknown exceeds the known available information.

\section{The Place of the RDN in Managing the CD Patients}

As mentioned above, the place of the RDNs in counseling, implementing, updating, anthropometric assessment and follow-up of the CD population is pivotal, indispensable and crucial among the team dealing with gluten-sensitive populations $[4,59,60,61,62]$. Their duties can be summarized, following CELIAC DISEASE letters*.

C- Consultation with a skilled dietitian

E- Education about CD

L- Lifelong adherence to a GFD

I- Identification and treatment of nutritional deficiencies

A- Access to an advocacy group

C- Continuous long-term follow-up

D- Dedication to the patient

I- Identification of local GFD facilities

S- Sources of hidden gluten

E- Extra-intestinal and complications knowledge

A-Amelioration of patients' quality of life

S- Support groups referral

E- Equilibrated and balanced diet

*Adopted from 14

\section{Conclusions}

Dietitians play a pivotal role in the education, follow-up and navigation of the GFD for patients affected by CD. Since gluten withdrawal is the cornerstone of CD therapy, and since various future therapeutic strategies, are not yet on the market, the patients relay heavily on the RDNs advice and service to cope with the GFD tough alley. Unfortunately, GFD nowadays represents also a torrid time. The actual surge in incidence, wheat content, gluten intake, CD-related T-cell stimulatory epitopes in wheat, usage in the processed food industries, nutritional deficiencies, changing phenotype and the fact that gluten is potentially detrimental to humankind health, make the RDNs role more complex, difficult but challenging. The 
most recent self-reported knowledge of the RDNs in GFD concluded that "RDNs may need more reinforcement on identification and treatment of nutritional deficiencies of CD" [5], but it seems that their role in the CD team is much vaster and GFD is a tough alley in a torrid time. As said, GFD for the RDNs dealing with CD is neither a solved problem nor a "closed case".

\section{References}

[1] Lerner A. New therapeutic strategies for celiac disease Autoimmun Rev. 2010;9:144-7.

[2] Tidwell D, Bomba A. Attitude of people with celiac disease towards dietitians and medical nutritional therapy. J Am Diet Assoc 2001; 101: A29.

[3] Mahadev S, Simpson S, Lebwohl B, Lewis SK, Tennyson CA, Green PH. Is dietitian use associated with celiac disease outcomes? Nutrients. 2013;5:1585-94

[4] Lamontagne P, West GE, Galibois I. Quebecers with celiac disease: analysis of dietary problems. Can J Diet Pract Res. 2001;62:175-81.

[5] Geiger J, Rhee Y, Stastny SN, Salafia EB. Celiac Disease and the Gluten-free Diet: Registered Dietitian Nutritionists' Self-reported Knowledge Varies. International J of Celiac Disease. 2017, 5(2).

[6] McNally SL, Donohue MC, Newton KP, Ogletree SP, Conner KK, Ingegneri SE, Kagnoff MF. Can consumers trust web-based information about celiac disease? Accuracy, comprehensiveness, transparency, and readability of information on the internet. Interact J Med Res. 2012;1:e1.

[7] Cameron BDMA (2008). Celiac Disease: Internet Resources.

[8] Stanley M. (2013). https://www.glutenfreeliving.com/gluten-freefoods/...gluten.../top-10-gluten-free-app....

[9] Guiu L. (2017). https://itunes.apple.com/us/app/the-gluten-freescanner-barcode.../id969482476?mt.

[10] aussiecoeliac (2015). www.aussiecoeliac.com.au/my-top-5-glutenfree-apps/.

[11] Megan (2012). thesethingsilove.com/2012/09/top-apps-forceliacs-and-gluten-free-diets/.

[12] Boyce B. Nutrition apps: opportunities to guide patients and grow your career. J Acad Nutr Diet. 2014;114:13-5.

[13] Meyer KG, Fasshauer M, Nebel IT, Paschke R. Comparative analysis of conventional training and a computer-based interactive training program for celiac disease patients. Patient Educ Couns. 2004;54:353-60.

[14] Niewinski MM.Advances in celiac disease and gluten-free diet. J Am Diet Assoc. 2008;108:661-72.

[15] Meeuwisse GW. Round table discussion. Diagnostic criteria in coeliac disease. Acta Paediatr. 1970;59:461-63.

[16] Ludvigsson JF, Leffler DA, Bai J et al. The Oslo definitions for coeliac disease and related terms. Gut. 2013 Jan; 62(1): 43-52.

[17] Picarelli A, Borghini R, Donato G, Di Tola M, Boccabella C, Isonne $\mathrm{C}$, Giordano $\mathrm{M}$, Di Cristofano $\mathrm{C}$, Romeo $\mathrm{F}$, Di Cioccio $\mathrm{G}$, Marcheggiano A, Villanacci V, Tiberti A. Weaknesses of histological analysis in celiac disease diagnosis: new possible scenarios. Scand J Gastroenterol. 2014;49:1318-24.

[18] Lerner A, Matthias T. Intraepithelial lymphocyte normal cut-off level in celiac disease: The debate continues. Internat $\mathrm{J}$ Celiac Dis.2016;4:4-6.

[19] Marsh MN. "It's Counting That Counts." Internation J of Celiac Dis 2016;4:1-3.

[20] Peña AS. Counting Intraepithelial Lymphocytes. Immunohistochemistry and Flow Cytometer are Necessary New Steps in the Diagnosis of Celiac Disease. Internation J of Celiac Dis. 2016;4:7-8.

[21] Akobeng AK, Thomas AG. Systematic review: tolerable amount of gluten for people with coeliac disease. Aliment Pharmacol Ther. 2008;27:1044-52.

[22] Gibert A, Kruizinga AG, Neuhold S, Houben GF, Canela MA, Fasano A, Catassi C. Might gluten traces in wheat substitutes pose a risk in patients with celiac disease? A population-based probabilistic approach to risk estimation. Am J Clin Nutr. 2013;97:109-16.

[23] Gibert A, Espadaler M, Angel Canela M, Sánchez A, Vaqué C, Rafecas M. Consumption of gluten-free products: should the threshold value for trace amounts of gluten be at 20, 100 or 200 p.p.m.? Eur J Gastroenterol Hepatol. 2006;18:1187-95.

[24] Collin P, Thorell L, Kaukinen K, Mäki M. The safe threshold for gluten contamination in gluten-free products. Can trace amounts be accepted in the treatment of coeliac disease? Aliment Pharmacol Ther. 2004;19:1277-83.

[25] Biagi F, Campanella J, Martucci S, Pezzimenti D, Ciclitira PJ, Ellis HJ, Corazza GR. A milligram of gluten a day keeps the mucosal recovery away: a case report. Nutr Rev. 2004;62:360-3.

[26] Collin P, Mäki M, Kaukinen K. It is the compliance, not milligrams of gluten that is essential in the treatment of celiac disease. Nutr Rev. 2004;62:490

[27] Lerner A, Jeremias P, Matthias T. Outside of Normal Limits: False Positive/Negative Anti TG2 Autoantibodies. Internat J Celiac Disease, 2015;3:87-90.

[28] Lauria L, Spinelli A, Cairella G, Censi L, Nardone P, Buoncristiano M; 2012 Group OKkio alla SALUTE. Dietary habits among children aged 8-9 years in Italy. Ann Ist Super Sanita. 2015;51:371-81.

[29] Lerner-Geva L, Bar-Zvi E, Levitan G, Boyko V, Reichman B, Pinhas-Hamiel O. An intervention for improving the lifestyle habits of kindergarten children in Israel: a cluster-randomised controlled trial investigation. Public Health Nutr. 2015;18:1537-44

[30] Eliyah Livshits O, Shauol R, Reifen R, Matthias T, Lerner A. Can Celiac Disease Present Along With Childhood Obesity? International Journal of Celiac Disease. 2017;5:19-23.

[31] Moreno LA, Gottrand F, Huybrechts I, Ruiz JR, González-Gross M, DeHenauw S; HELENA Study Group. Nutrition and lifestyle in European adolescents: the HELENA (Healthy Lifestyle in Europe by Nutrition in Adolescence) study. Adv Nutr. 2014; 5: 615S-623S

[32] Olsson C, Hörnell A, Ivarsson A, Sydner YM. The everyday life of adolescent coeliacs: issues of importance for compliance with the gluten-free diet. J Hum Nutr Diet. 2008; 21:359-67.

[33] Casella S, Zanini B, Lanzarotto F, Villanacci V, Ricci C, Lanzini A. Celiac disease in elderly adults: clinical, serological, and histological characteristics and the effect of a gluten-free diet. J Am Geriatr Soc. 2012; 60:1064-9.

[34] Vilppula A, Kaukinen K, Luostarinen L, Krekelä I, Patrikainen H, Valve R, et al. Clinical benefit of gluten-free diet in screendetected older celiac disease patients. BMC Gastroenterol. 2011; 11:136.

[35] Lerner A, Matthias T. Increased knowledge and awareness of celiac disease will benefit the elderly. Intern. J of Celiac Dis. 2015 3:112-114.

[36] Lerner A, Jeremias P, Matthias T. The world incidence and prevalence of autoimmune diseases is increasing: A review. Internat J Celiac Disease. 2015;3:151-155.

[37] Lerner A, Jermias P, Matthias T. The world incidence of celiac disease is increasing: a review. Internat. J. Of Recent Scient. Res. 2015;7:5491-5496.

[38] Lerner A, Matthias T. Possible association between celiac disease and bacterial transglutaminase in food processing: a hypothesis. Nutr Rev. 2015;73:544-552.

[39] Lerner A, Matthias T. Changes in intestinal tight junction permeability associated with industrial food additives explain the rising incidence of autoimmune disease. Autoimmun Rev. 2015;14:479-89.

[40] Matthias T, Jeremias P, Neidhöfer S, Lerner A. The industrial food additive microbial transglutaminase, mimics the tissue transglutaminase and is immunogenic in celiac disease patients. Autoimmun Rev, 2016;15:1111-1119.

[41] Lerner A, Matthias T. Multiple food additives enhance human chronic diseases. SOJ Microbiology\&Infectious Dis. 2016;4:1-2.

[42] Lerner A, Matthias T. Are Microbial Enzymes Used Safe in the Processed Food Industries? Food and Bioprocess Technology, 2016;9: 2125-2126

[43] Lerner A, Matthias T. Don't forget the exogenous microbial transglutaminases: it is immunogenic and potentially pathogenic. Biophysics, 2016;3:529-535.

[44] Lerner A, Aminov R, Matthias $T$. Intestinal dysbiotic transglutaminases are potential environmental drivers of systemic autoimmunogenesis. Frontiers in Microbiology, 2017;8;article 66.

[45] Lerner A, Matthias T. Extraintestinal manifestations of CD: Common pathways in the gut-remote organs' axes. Internat $\mathrm{J}$ Celiac Dis. 2017;5:24-27. 
[46] Van den Broeck HC, de Jong HC, Salentijn EMJ, et al. Presence of celiac disease epitopes in modern and old hexaploid wheat varieties: wheat breeding may have contributed to increased prevalence of celiac disease. Theor Appl Genet. 2010;121:1527-39.

[47] Molberg O, Uhlen AK, Flaete NS, et al. Mapping of gluten T-cell epitopes in the bread wheat ancestors: implications for celiac disease. Gastroenterology 2005;128:393-401.

[48] Lerner A. Balanced polymorphism: a survival advantage in celiac disease. Editorial. Med Hypotheses, 2011;77:1-2.

[49] Lerner A. The last two millennias eco-catastrophes are the driving forces for the potential genetic advantage mechanisms in celiac disease. Med Hypotheses. 2011;77:773-6.

[50] Greco L. From the neolithic revolution to gluten intolerance: benefits and problems associated with the cultivation of wheat. Pediatr Gastroenterol Nutr. 1997;24:S14-6.

[51] Branski D, Ashkenazy A, Freier S, Lerner A, Dinari G et al. Extraintestinal manifestations and associated disorders of celiac disease. In: "Gluten-Sensitive Enteropathy". Front Gastrointest Res. Eds. Branski D, Rozen P, Kagnoff MF. Karger, Basel. pp. 164-175. 1992.

[52] Lauret E, Rodrigo L. Celiac Disease and Autoimmune-Associated Conditions Biomed Res Int. 2013; 2013: 127589.

[53] Cárdenas-Roldán J, Rojas-Villarraga A, Anaya JM. How do autoimmune diseases cluster in families? A systematic review and meta-analysis BMC Med. 2013; 11: 73 .
[54] Haimi M, Lerner A. Nutritional Deficencies in the pediatric age group in a multicultural developed country- Israel. World Journal of Clinical Cases. 2014;2:120-125.

[55] Lerner A, Gruener N, Iancu TC. Serum carnitine levels in coeliac disease. Gut 1993; 34:933-935.

[56] Lerner A, Shapira Y, Agmon-Levin N, , Pacht A, Ben-Ami Shor D, López Hoyos M, Sanchez-Castanon M, Shoenfeld Y. The clinical significance of $25 \mathrm{OH}$-vitamin D status in celiac disease. Crit Rev Allerg Immunol. 2012;42:322-330.

[57] Abbasi N, Allameh SF. Extra intestinal manifestations of celiac disease and associated disorders. Internat J Celiac Dis. 2017; 5:1-9.

[58] Lerner A, Matthias T. Extraintestinal manifestations of CD: Common pathways in the gut-remote organs' axes. Internat $\mathrm{J}$ Celiac Dis. 2017;5:24-27.

[59] Case S. The gluten-free diet: How to provide effective education and resources. Gastroenterol 2005;128:s128-134.

[60] Theethira TG, Dennis M. Celiac disease and the gluten-free diet: consequences and recommendations for improvement. Dig Dis. 2015;33:175-82.

[61] Kupper C. Dietary guidelines and implementation for celiac disease. Gastroenterology. 2005;128(4 Suppl 1):S121-7.

[62] Pietzak MM. Follow-up of patients with celiac disease: achieving compliance with treatment. Gastroenterology. 2005; 128 (4 Suppl 1) S135-41. 